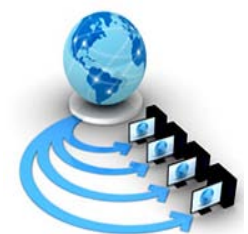

Volume 9, No. 5, September-October 2018

\title{
ALL TERRAIN ROBOTIC VEHICLE WITH ROBOTIC ARM FOR DANGEROUS OBJECT DISPOSAL
}

\author{
Vidyashree $\mathrm{H}$ \\ Electronics and Communication Engineering, \\ Global Academy of Technology \\ Bangalore, India
}

\section{Umesh S}

Computer Science Engineering, Dr. Ambedkar Institute of Technology

Bangalore, India

\author{
Chaithra K B \\ Computer Science Engineering, \\ Dr. Ambedkar Institute of Technology \\ Bangalore, India
}

Nagamayuri B S

Information Science Engineering, Jyothi Institute of Technology

Bangalore, India

\begin{abstract}
The Robotics Technology is now rapidly emerging in field of engineering as they are capable of carrying out the complex sets of action automatically higher accuracy and speed. In this project, the robotic vehicle is designed to pick and place the object with a 2-degree motion robotic arm for safety reasons. The vehicle is android application controlled for remote operations via Bluetooth. Bluetooth device is connected to the microcontroller to drive DC motors via motor driver IC for necessary operations. Remote operation can be accomplished by any smartphone/tablet etc., with Android Operating System; upon a GUI (Graphical User Interface) based touch-screen operation. The Rocker-Bogie method is implemented for the easier movement of the robot on all the terrain surfaces. The metal detector is used to detect any metals on the surface.
\end{abstract}

Keywords: 2-degree motion, Remote Operation, Bluetooth Device, Metal Detector, Rocker-Bogie method.

\section{INTRODUCTION}

Robots are indispensible in almost all the industries now a day. This is mainly due to its ability to perform functions with higher accuracy and speed than that of the most experienced human operator. Robots are usually programmed to be job specific unlike humans who can any time switch tasks and be more versatile which can be overcome in future with the flourishing technology. These robots interact with the physical world via sensors and actuators. Robots can replace humans in some hazardous duty service without risking human lives. The nuclear industry was a first to develop and use robotic arms for handling radioactive materials. Robotic arms allowed scientists to be located in clean, safe rooms operating controls for the robotic arms located in radioactive rooms.

In this highly advancing society, time and man power are severe constraints for completion of task in large scales. The automation can help in repeating frequently carried tasks without getting tired. One of the major performed tasks is picking and placing of jobs from one place to other. The pick and place robot is a microcontroller based mechatronic system that detects the object, picks that object from source location and places it at desired location. For detection of metal, metal detectors are used which detect presence of metal as the magnetic fields in the coil of metal detector is interrupted by placed metal.

Use of sophisticated objects, devices and appliances never imagined that the modern-day technology has made available. The weapons mostly used for attacks are bombs and improvised explosive devices which have been responsible for the loss of many lives. Moreover, military personnel take risk while on reconnaissance missions.
Nuclear experts are firefighting; teams are not left out as they are involved in handling hazardous or radioactive materials and bare the risks while accessing deadly fire scenes. This has prompted the need for the development of a robotic arm which can used to dispose dangerous objects such as bombs or explosives before they explode; thereby, protecting lives and properties. These robots can also work in the environment which is hazardous to the human lives. The system is triggered by a remote control thereby contributing a line of defense to the lives and preventing loss of proven and tested personnel.

\section{METHODOLOGY}

The method employed in designing and constructing a robotic arm are based on the operational characteristics and features of the microcontrollers, stepper motors, servo motors and programming of microcontroller, stepper and servo motors. The robot was designed for picking and placing the object. The robot is made to move on all terrain surfaces by implementation of Rocker-Bogie method.

The Bluetooth module HC-05 is used for a communication between android application which acts as a remote control and the ATmega328 microcontroller. The android application is used to send the control signal to a Bluetooth device which in turn sends it to the microcontroller. The microcontroller then receives and passes the control signal and sends the command to the motor driver to drive the motors in the required direction. The power supply of $12 \mathrm{v} \mathrm{DC}$ is given and a separate $9 \mathrm{v}$ battery is given for metal detector. Three servo motors are used for designing a robotic arm with 2-degree motion. Two servo motors are used for upward and downward 
movement of the robotic arm whereas, one servo motors for the gripper movement.

\section{BLOCK DIAGRAM}

The block diagram of the built system is shown in Figure 1. It consists of an ATmega328 microcontroller IC, Bluetooth module, Six DC motors and three servo motors with driver IC and power supply. The pick and place robotic arm consists of a robotic arm placed on the moving vehicle. The vehicle can move along any type of surface irrespective of it is smooth or rough. It is implemented with Rocker-Bogie method for the smooth and reliable operation. The pick and place robot uses nine motors for the operation of the system, Six DC motors for the operation of the vehicle movement and three servo motors for the pick and place operation. The robotic arm consists of an arm assembly with a jaw, which is only able to move in up and down direction.

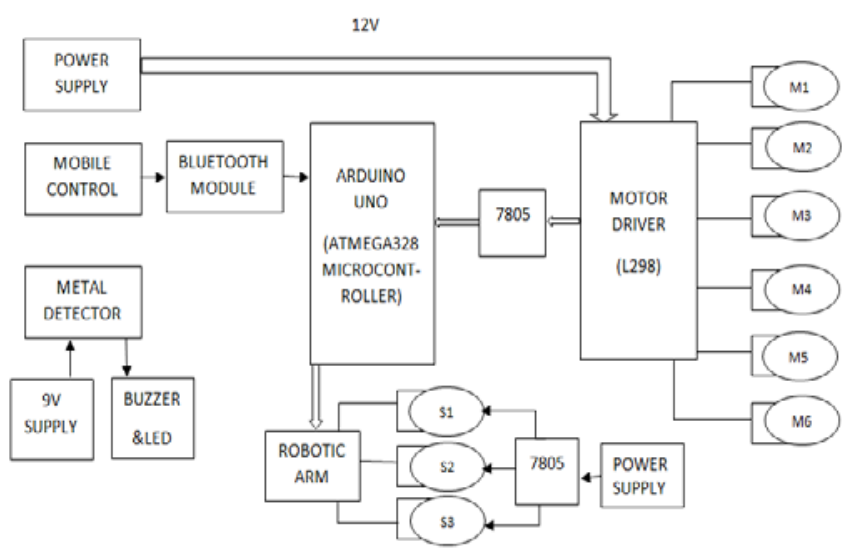

Figure 1: Block Diagram of Pick and Place Robot

\section{ROCKER-BOGIE METHOD}

The Rocker-Bogie mechanism is the most popular linkage mechanism for a wheel robot which has six actuated wheels. The Rocker-Bogie's main frame has two linkages on each side that are called the 'rocker or rover'. One end of the rocker is connected to a small linkage which is called "Bogie". The Bogie relates to the front wheel and the middle wheel. The rocker and the bogie relate to the passive joint, so that the six wheels contact the ground always without any actuators.

To move the robot on all the terrain surfaces and makes it able to climb stairs of about 5 to $8 \mathrm{cms}$, Rocker-Bogie method was implemented. This method has two parts i.e., Rover and Bogie. Rover is a vehicle for driving over rough terrain especially one driven by remote control over extra-terrestrial terrain. Robotic rovers are used broadly for exploratory and investigation purposes in the fields of scientific exploration and defense. The rover will be having a Rocker-Bogie Suspension system with six wheels for greater stability in maneuvering over obstacle. It can avoid an un-mountable obstacle and can traverse over mountable obstacles. Instructions are sent to the rover by a remote control connected over Bluetooth. When one rocker goes up the other goes in the reverse direction. The chassis prolongs the common pitch angle of both rockers. One end of a rocker is fitted with the drive wheel and the other end is pivoted to a Bogie. The term "Bogie" refers to the links that have a drive wheel at the same end. The suspension has the six wheels with the symmetric structure for both sides. Each side has three wheels which are connected to each other two, with links. The main linkage called rocker has two joints while first joint is connected to front wheel, the other joint is assembled to another linkage called bogie, which is like train wagon suspension member.

\section{BLUETOOTH COMMUNICATION}

To design a HC-05 Bluetooth based Android Mobile controlled wireless robot with AVR Atmega328 microcontroller. Here, we will use the Android mobile as the input device to drive the motor in different direction. The communication between HC-05 Bluetooth Module and Android Mobile takes place through wireless Bluetooth technology and the communication between HC-05 Bluetooth module and Atmega328 Microcontroller takes place through UART serial communication protocol.

The HC-05 Bluetooth module and the Android Mobile are connected through Bluetooth. End user enters the input signal from the associated Android Mobile through Bluetooth terminal app and the Android Mobile transmits the control signal to the HC-05 Bluetooth module through its Bluetooth. The HC-05 Bluetooth module receives the control signal and transmits it to the Atmega328 microcontroller through UART. The microcontroller receives the control signal and processes it and sends the required control signal to the DC Motor Driver (L298) of the robot to drive the robot in the desired direction and it will send direction of movement of robot to Android mobile also.

The control keys for the robot are forward, backward, left, right, and stop to drive the robot in respective directions and to stop it respectively. Similarly, servo1/2+ for arm up and servo 1/2- for arm down and grip+ for arm open and grip- for arm close. Now press different keys from the Android Mobile drive your robot as you wish.

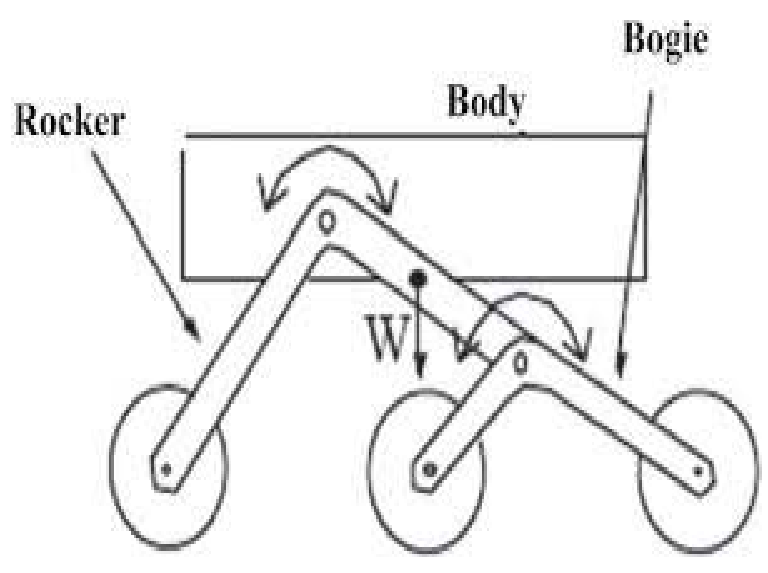

Figure 2: Rocker-Bogie Suspension,

\section{SYSTEM DESIGN}

For the controlling of motor, Motor and Driver IC Atmega328 microcontroller is used. The input signal or the control signal is given from an Android device, which is interfaced with the microcontroller via Bluetooth module. L298 has two sets of arrangements where one set has input1, 2 and output 1, 2 and the other set has input 3, 4 and output 3,4. The program is so written i.e., while executed it sends commands to the Motor 
Driver IC as per its requirements for running the motor for the movement of the robot. The Android application is used for sending commands for left, right, forward and backward movement and to stop the robot when required.

$12 \mathrm{~V}$ battery powers the circuit in series with regulator IC LM7805 for the microcontroller which are standard connections like crystal, reset arrangement indication LED etc., A Bluetooth device being powered from a reversed biased Zener diode is interfaced to the microcontroller that after being paired with any smartphone communicates with this Bluetooth for taking appropriate actions as per the touch operation made on the smart phone. The work uses servo motors working on similar technology for the arm up and down/open and close duly interfaced to the microcontroller with duly pulled resistors. The Android application utilized in this project is" Bluetooth joys”.

The program is so written that for the touch screen operation from the smartphone results in command being sent through the Bluetooth module, on grip $+=$ open, grip- = close, servo1+/servo2+ = Up and servo1-/servo2 =Down rotation of the motor which can be seen in Figure 3.
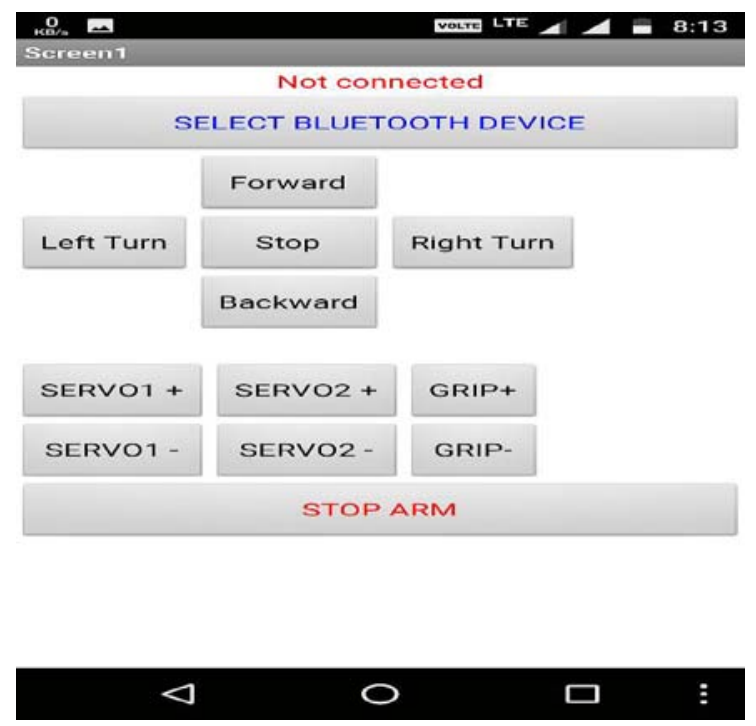

Figure 3: Blue-control/Android Application.

\section{2-DEGREE MOTION}

The degrees of freedom specify the modes in which the robotic arm or a system can be moved. The 2- Degree motion has two natural frequencies and for each natural frequency there corresponds a natural state of vibration with a displacement configuration known as the normal mode.

Here, moving the robotic arm either upward and downward or left and right contributes to 2-Degree Motion which in turn means to enable the movement in two directions.

\section{APPLICATIONS}

1. Military Applications: It can be used to discard bombs and other explosives and can also be used for various shipments.

2. Industrial Applications: Robots can be used as sweepers, welders etc.
3. Medical Applications: Robots can be used to lift the required equipment and in future it can also perform many minor or major operations.

4. Space Applications: It can be used to space exploration.

\section{RESULTS}

The Figure 3 and 4 shows the resulted hardware model after the completion of the model.

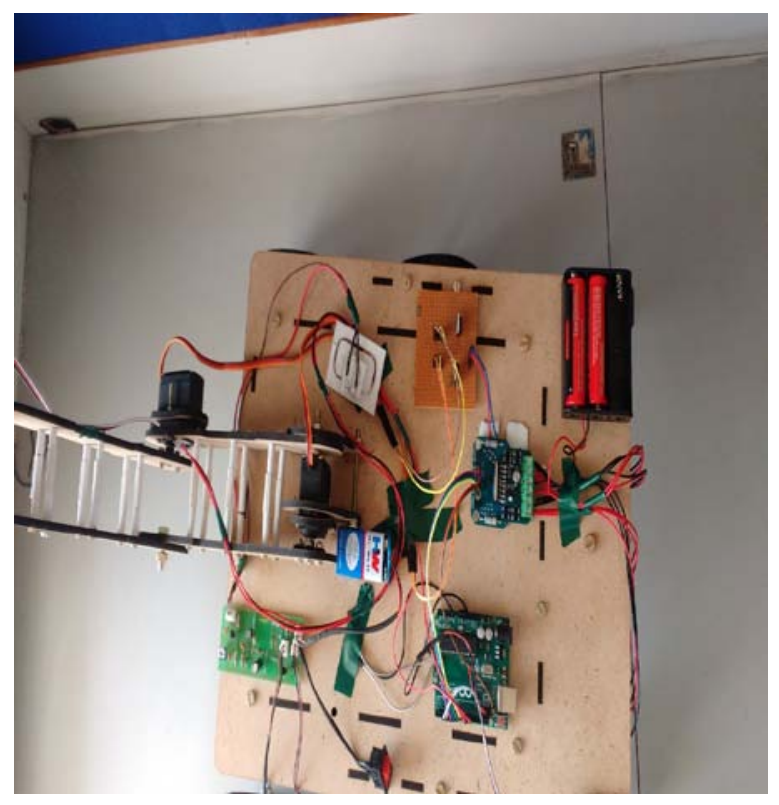

Figure 4: Top-view of the Robot

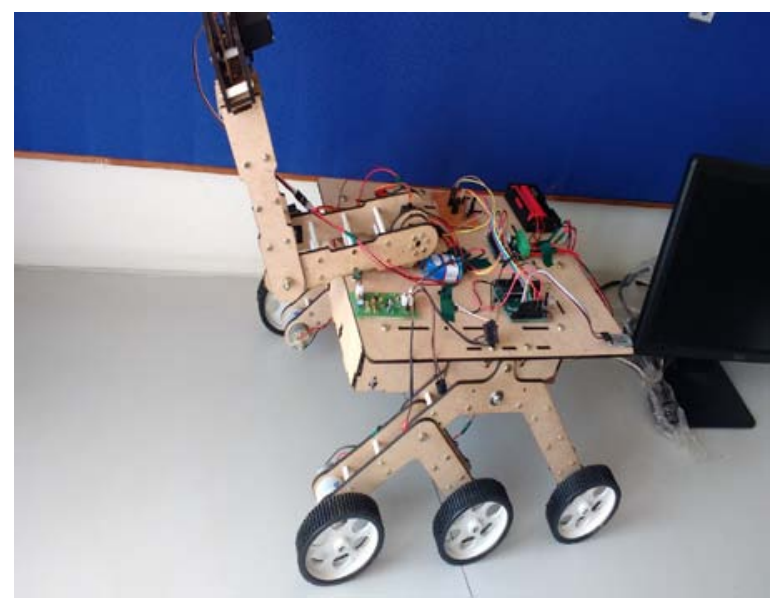

Figure 5: Hardware Model

\section{CONCLUSION AND FUTURESCOPE}

The terrain rover with Rocker-Bogie suspension is completed successfully and its operation is tested and found to be correct. The paper gives the step by step procedure that is to be followed which resulted in a robotic research platform. The main feature of this is a soft catching arm. We know that, when handling the explosive item like bomb it will be handled smoothly. The maximum weight that can be carried by this model depends on the capacity of the DC motors used. 
1. The robot can be upgraded by interfacing with wireless cameras, night-vision cameras, explosive detectors etc. for finding the bombs and proper vision assistance.

2. The power Limitations can be overcome by using the solar panels to charge the batteries.

3. Sensors can be used to automatically detect the obstacles and stop to weep buzzer for notifying which in turn can avoid accidents.

\section{REFERNCES}

[1] John Lovine, "Robots Androids, and Animations 12 Incredible Projects You can Build”,[1] Second Edition, McGraw-Hill.2002.

[2] Sungwookmoon, Yung Jinkim, Hojunmyeong, Changsookim, Namjucha, and Dong Hwankim.”Implementation of SmartPhone Environment Remote Control and Monitoring System for Android Operationg System based Robot Platform”,[2] IEEE Robot Automation Mag. Volume 13, no.4, pp.82-90,Dec.2006

[3] Mohamed Naufal Bin Omar "Pick and Place Robotic Arm Controlled by Computer”,[3] Universititeknikal Malaysia, Melaka Apr 2007.

[4] K.Harada "Grasp Planning for Parallel Grippers with Flexibilty on it's Grasping Surface”,[4] Proc. Of IEEE International Conference on Robotics and Biomimetics,2011.
[5] C. Borst, M.Fischer, G. Hirzinger,[5] "Fast-Robust Grasping Plan for Arbitary 3D Objects".

[6] V. Lippiello, F. Ruggiero, V.Sieiliano, and L. Villanei, [6] "Visual Grasp Planning for unknown Objects using Robotic Hand which is multi-figured".

[7] E. Malis, F. Chaumette, and S. Boudet, "Visual servoing"IEEE Transaction Robot Automation,[7] Volume 15, No. 2 , pp.238-250,Apr1999.

[8] A.T. Miller "Automatic Grasp Planning using Shape Primitives", Proc. IEEE International Conference on Robotics and Automation, [8] pp.14-19,2003.

[9] K. Nagatha "To Pick up an indicated Object from a Complex Environment”,[9] Proc. IEEE/RSJ International Conference on Robots and Systems|pp. 2109-2116, 2010

[10] D. Berenson, J. Kuffner, and H. Choset, "Optimzation Approach to Plannig Mobile Manipulation” [10] Proc. Of IEEE International Conference on Robotics and Automation, pp.1187-1192, 2008.

[11] SYJuang, JG Juang.[2012] "Real time indoor surveillance based on smartphone and mobile robot" [11] $10^{\text {th }}$ IEEE International Conference on Industrial Information(INDIN), Beijing, 475-480.

[12] Vivek Ramakrishan, Nalamwar Sanchit Gopal, Rahul Ashok and S.Moorthi, "FPGA based DC Servo Motor Control for Remote Pelication of Movements of Surgical Arm” [12], 978-1-4577-0255-6/11/2011 IEEE. 